\title{
On the Use of the Platelet Activity State Assay for the In Vitro Quantification of Platelet Activation in Blood Recirculating Devices for Extracorporeal Circulation
}

\author{
*Filippo Consolo, *Lorenzo Valerio, †Stefano Brizzola, *Paolo Rota, *Giulia Marazzato, \\ $*$ Valentina Vincoli, $\ddagger$ Stefano Reggiani, *Alberto Redaelli, and *Gianfranco Fiore \\ *Dipartimento di Elettronica, Informazione e Bioingegneria, Politecnico di Milano; $†$ Dipartimento di Scienze Veterinarie \\ per la Salute, la Produzione Animale e la Sicurezza Alimentare, Facoltà di Medicina Veterinaria, Università di Milano, \\ Milano; and $\$$ Sorin Group Italia, Mirandola, Italy
}

\begin{abstract}
We designed an experimental setup to characterize the thrombogenic potential associated with blood recirculating devices (BRDs) used in extracorporeal circulation (ECC). Our methodology relies on in vitro flow loop platelet recirculation experiments combined with the modified-prothrombinase platelet activity state (PAS) assay to quantify the bulk thrombin production rate of circulated platelets, which correlates to the platelet activation (PA) level. The method was applied to a commercial neonatal hollow fiber membrane oxygenator. In analogous hemodynamic environment, we compared the PA level resulting from multiple passes of platelets within devices provided with phosphorylcholine (PC)-coated and noncoated (NC) fibers to account for flow-related mechanical factors (i.e., fluid-induced shear stress) together with surface contact activation phenomena. We report for the first time that PAS assay is not significantly
\end{abstract}

sensitive to the effect of material coating under clinically pertinent flow conditions $(500 \mathrm{~mL} / \mathrm{min})$, while providing straightforward information on shear-mediated PA dynamics in ECC devices. Being that the latter is intimately dependent on local flow dynamics, according to our results, the rate of thrombin production as measured by the PAS assay is a valuable biochemical marker of the selective contribution of PA in BRDs induced by device design features. Thus, we recommend the use of PAS assay as a means of evaluating the effect of modification of specific device geometrical features and/or different design solutions for developing ECC devices providing flow conditions with reduced thrombogenic impact. Key Words: Platelet activation-Blood recirculating devices-Shear- and contact-associated thrombogenicity—Extracorporeal circulation.
Open heart surgery involving cardiopulmonary bypass $(\mathrm{CPB})$ represents a highly thrombogenic clinical environment (1). The extracorporeal circulation (ECC) regimen induces marked abnormalities of hemostasis, a potentially fatal complication when associated with congenital and/or acquired coagulation disorders (2). Thromboembolic events are primarily caused by the combination of two effects which contribute in enhancing platelet activation (PA) leading to thrombus formation: (i) the mechanical contribution upon the flowing corpuscu-

\section{doi:10.1111/aor.12672}

Received July 2015; revised September 2015.

Address correspondence and reprint requests to Dr. Filippo Consolo, Dipartimento di Elettronica, Informazione e Bioingegneria, Politecnico di Milano, Piazza Leonardo da Vinci 32, Milano, MI 20133, Italy. E-mail: filippo.consolo@polimi.it lar blood elements, and (ii) the contact of blood cells with the nonendothelial surfaces of the ECC circuit tubing and of the blood recirculating devices (BRDs, e.g., blood oxygenator, heat exchanger, arterial filter, etc.) $(1,3)$. Mechanical solicitation is due to the synergistic action offered by the propelling devices (the pump) and the nonphysiologic flow conditions causing abnormal shear stress patterns within the ECC circuit and the BRD geometry. In particular, $\mathrm{PA}$ in BRDs is considered as one of the major precursors of the onset of thrombosis and thromboembolism during the surgery. The risk of PA is not totally eliminated by administering antithrombotic drugs, which, in turn, may induce further complications such as iatrogenic hemorrhage and postoperative bleeding vulnerability (4). So far, the problem of thrombosis during CPB remains a concern even in the presence of surface modification/coating strate- 
gies (5), suggesting that flow-related phenomena, that is, the hemodynamic-related shear stress patterns experienced by platelets within specific device geometrical features, prevail in triggering PA. However, at present, there is a lack of studies investigating the pro-coagulant properties of platelets induced by flow through these devices, and the definition of a systematic strategy to optimize the design of BRDs, that is, to reduce or eliminate flow conditions that cause PA, still needs to be addressed.

Recently, research studies examining the impact of implantable mechanical circulatory support (MCS) devices on PA and thrombosis led to the development of the modified prothrombinase platelet activity state (PAS) assay. The PAS assay is a laboratory method able to measure the near real-time rate of formation of thrombin-a universal thrombogenic marker-by activated platelets and to correlate with their activation level (6). The test is performed with gel-filtered platelets (GFPs, i.e., platelets deprived of plasma proteins), and the assay makes use of acetylated prothrombin (Ac-FIIa) as the thrombin substrate (7). The PAS assay allows measuring the prothrombotic tendency of activated platelets, that is, the platelet procoagulant activity, by detecting the thrombin production rate of activated platelets, without inducing aggregation or clot formation during the test. Mechanisms leading to platelet aggregation are precluded during the test, mainly due to the removal of plasma coagulation factors (mediating aggregation in whole blood) from GFP preparations (7). Furthermore, Ac-FIIa, while retaining the ability to produce thrombin upon activation by GFP-bound prothrombinase complex, is characterized by low $(<0.1 \%)$ clotting activity ( 7$)$. Moreover, the thrombin produced by Ac-FIIa is inactive on fibrinogen (7) and thus cannot mediate the conversion of fibrinogen to fibrin. In addition, the use of Ac-FIIa allows inhibiting the physiologic thrombinmediated positive feedback on PA. In detail, the thrombin produced by the conversion of Ac-FIIa does not feed back on the factor Xa (FXa) complex to further activate platelets (7). Hence, with PAS assay, the dose- and time-PA dynamics can be characterized, in response to differing levels (i.e., varying the dose and/or the exposure time) of the agonist, ensuring a one-to-one relationship between the inducer and the actual level of PA, which is proportional to the thrombin production rate.

The PAS assay has been utilized extensively for the in vitro characterization of shear-dependent PA in MCS devices, such as prosthetic heart valves (8-13), ventricular assist devices $(6,14,15)$, and the total artificial heart $(16,17)$, where the nonphysiological blood flow patterns (i.e., pulsatility, eddy formation, turbulences, rapid flow acceleration/ deceleration phase, etc.) predominate the platelet response. The PAS assay has been exploited through two different approaches. It has been used as the final step of an advanced design methodology, known as device thrombogenicity emulation (DTE) (18-20). This approach couples in silico analysis, to extract device-specific flow conditions in the numerical virtual domain, with in vitro experiments; specifically, selected most critical shear stress patterns are replicated on platelet samples through an experimental apparatus, named hemodynamic shearing device (19), emulating the predicted flow conditions; finally, the related thrombogenic potential is quantified through the PAS assay. The DTE is a straightforward process to optimize the thrombogenic performance of devices by estimating the effects of modifications of specific design parameters in terms of devicerelated hemodynamics. However, such an approach cannot account for the whole fluid dynamics but can estimate the potential PA on selected platelet trajectories; as a matter of fact, it can be extremely useful in the preliminary phase of BRD design. It is worth noticing that it neglects the contact-associated PA. Alternatively, the PAS assay has been used for characterizing the dynamics of PA within flow loop systems, where platelets are recirculated within physical prototypes of the investigated devices and thrombogenicity is measured in timed samples extracted from the recirculation loop. When used in combination with flow loop systems, the PAS assay becomes extremely useful in an advanced stage of BRD design, providing an accurate assessment of the PA device potential. In fact, it allows to comprehensively characterize the hemodynamic-related thrombogenic potential of the device. Moreover, it accounts, in principle, for both PA mechanisms, including contact-associated PA, which could be particularly critical in BRDs, such as oxygenators, which are characterized by considerable surface area and higher platelet residence time with respect to MCS.

With this work, we aimed at investigating the potential of extending the use of PAS assay to comprehensively characterize PA in BRDs that are used in ECC procedures. To this purpose, an experimental platform was developed to circulate platelets within a commercial hollow fiber oxygenator and to quantify the dynamics of PA via the PAS assay. Flow loop experiments were performed replicating clinical-like perfusion conditions, and the proposed methodology was applied to devices with phosphorylcholinecoated (PC) fibers and non-coated (NC) devices. In this way, we tested the capability of the PAS assay (i) 
to quantify the shear-induced activation of platelets in ECC devices, where shear loads are mainly determined by flow through a fiber mat, and (ii) to evaluate at what extent the PAS assay is sensitive to the contribution offered by platelet surface contact interactions. This work represents a first step toward the establishment of a laboratory platform able to predict and prospectively limit the impact of thrombogenicity associated to ECC practice.

\section{MATERIALS AND METHODS}

\section{The hollow fiber blood oxygenator}

The Dideco Kids D100 neonatal perfusion support system (Sorin Group Italia S.r.l., Mirandola, Italy) was used in our experimental campaign. The device has a static priming volume of $31 \mathrm{~mL}$ and is equipped with: (i) a microporous hollow fiber (polypropylene) bundle oxygenator (OXY) characterized by a $0.22-\mathrm{m}^{2} \mathrm{mem}-$ brane surface area for gas transfer, and (ii) a metal coil (stainless steel) heat exchanger (HE) with a $0.03-\mathrm{m}^{2}$ surface area to set and control the patient's blood temperature during the surgery. In the device, the blood circumferential flow path is established (21).

\section{Preparation of platelet samples}

Whole blood $(250 \mathrm{~mL})$ was drawn through venipuncture from living adult ovine, collected into a blood bag (TERUFLEX, Terumo Corporation, Tokyo, Japan) containing $35 \mathrm{~mL}$ of citrate phosphate dextrose adenine (CPDA-1) as anticoagulant and transferred to the laboratory. Purified GFPs were prepared from whole blood as described in Sheriff et al. (6) (Fig. 1) and diluted to a standard concentration of 20000 platelets/ $\mu \mathrm{L}$ in a HEPES-modified $\mathrm{Ca}^{2+}$-free Tyrode's buffer (Platelet Buffer) containing $0.1 \%$ fatty acid-free bovine serum albumin, with $3 \mathrm{mM} \mathrm{Ca}^{2+}$ added $10 \mathrm{~min}$ prior to experiments $(7,22)$. Equilibrated GFPs were maintained with gentle agitation at room temperature and used for flow loop experiments within $6 \mathrm{~h}$ of gel filtration.

\section{Flow loop circulation setup}

An experimental setup for flow loop GFP circulation experiments was designed (Fig. 2) including (i) a 50-mL Falcon tube (PBI, VWR International PBI S.r.l., Milan, Italy) serving as GFP reservoir immersed into a water-bath (VWB 6, VWR International) to set the desired GFP temperature $\left(37^{\circ} \mathrm{C}\right)$ during the test; (ii) a roller pump (Stockert-Shiley 10-10-00, Stockert GmbH, Freiburg, Germany) to set the desired GFP flow perfusion rate (Q); (iii) a flow meter (HT110, Transonic Systems Inc., Ithaca, NY, USA) equipped with a clamp-on flow probe (H6XL, Transonic); and (iv) two pressure sensors (PRESS-S000, PendoTECH, Princeton, NJ, USA) to monitor the $\mathrm{Q}$ and the pressure drop $(\Delta \mathrm{P})$ across the OXY, respectively; $\mathrm{Q}$ and $\Delta \mathrm{P}$ were continuously monitored during the test and acquired via an $\mathrm{I} / \mathrm{O}$ hardware and a custom-made software interface (DAQcard-6036E and LabView, National Instruments, Austin, TX, USA). The setup had a total priming volume of $95 \mathrm{~mL}$ (Kids D100 [31 mL] + hydraulic circuit [57 mL] + GFP volume-reservoir [7 mL]). In Table 1, dimensions and materials of the fluidic circuit components are summarized. For each experiment, a new hydraulic circuit was used.

\section{Flow loop experiments}

In Fig. 3, a schematic of the circulation flow loop is provided. After the hydraulic circuit was gently filled with GFP and accurately debubbled, the pump was operated and GFP circulated for $120 \mathrm{~min}$, which corresponded to more than 600 fluid passes through the device, on average (Table 2). A Q equal to $500 \mathrm{~mL} /$ min was set, corresponding to the average value of the operating range recommended by the manufacturer for the Kids D100 (23). In the performed experiments, the HE was not supplied with heating fluid, and no gas was supplied through the gas inlet port of the OXY module, so as to prevent heat and gas exchange phenomena to influence the platelet response. At pre-determined time-steps (Table 2)

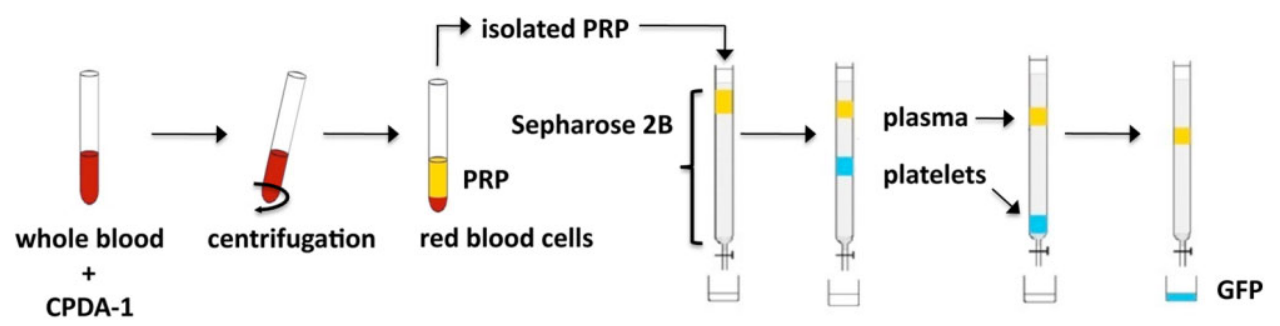

FIG. 1. Schematic of the procedure for GFP extraction: whole blood was centrifuged $(500 \times g$ for 15 min) to obtain platelet-rich plasma (PRP); PRP was isolated from red blood cells and gel-filtered through a column of Sepharose 2B (GE-Healthcare Life Sciences) to separate plasma proteins from collected gel-filtered platelets (GFP). 


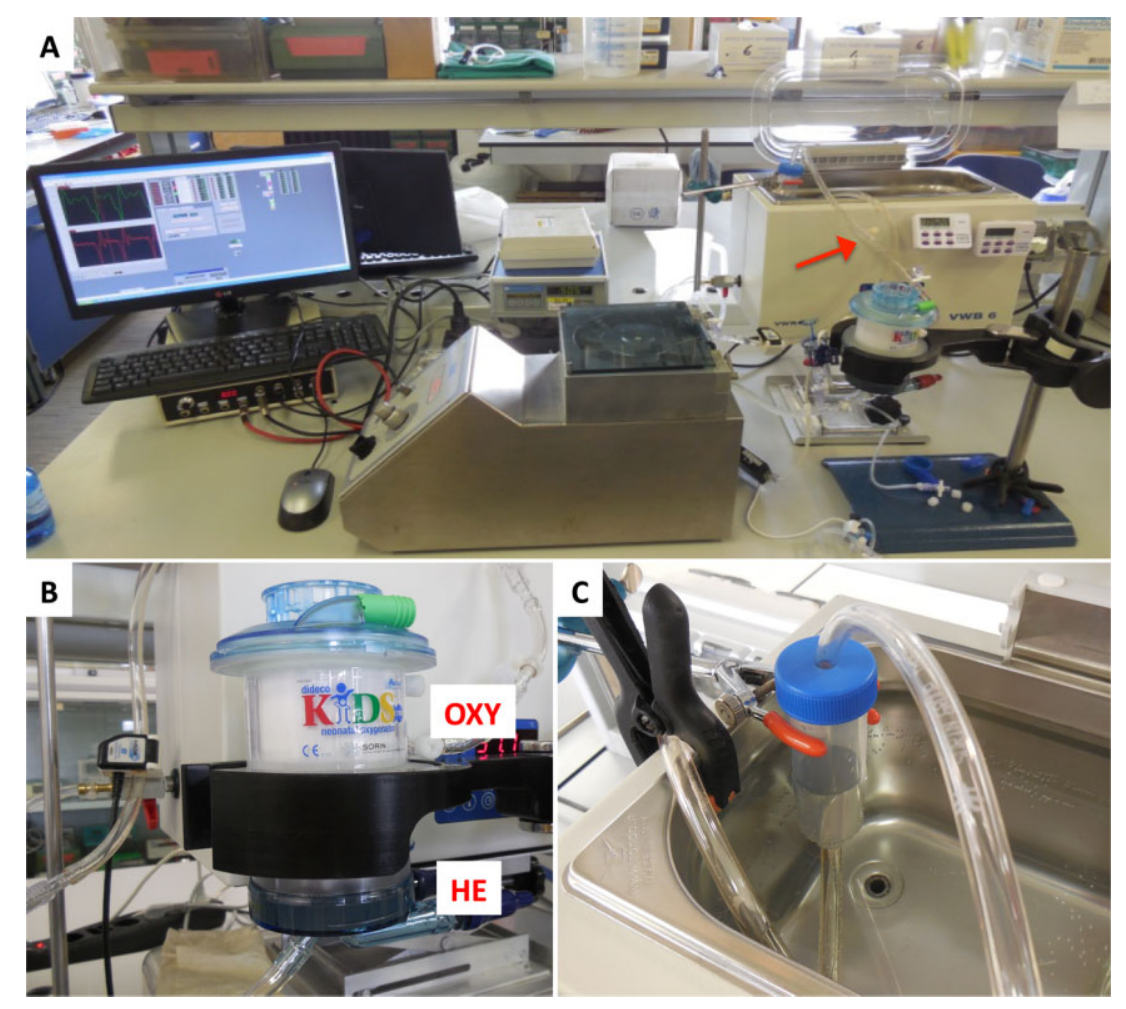

FIG. 2. (A) The experimental setup used for flow loop GFP circulation experiments: platelets were circulated for $120 \mathrm{~min}$ at a $500-\mathrm{mL} / \mathrm{min}$ flow rate; a GFP sampling port was provided downstream of the Kids D100 (red arrow): sampled platelets were assayed via the PAS assay. (B) The metallic heat exchanger (HE) and the polymeric hollow fiber bundle oxygenator (OXY) modules comprising the Kids D100 neonatal perfusion system. (C) The Falcon tube serving as GFP reservoir.
GFP samples were taken and assayed for PA via the PAS assay; a disposable $1-\mathrm{mL}$ syringe with an $18 \mathrm{G} \times$ $1 / 2^{\prime \prime}$ needle (Terumo Corporation) connected to a stopper connector (CareFusion Ltd., Basingstoke, UK) was used to access flowing GFP (Fig. 3). For each GFP sampling, a new syringe was used.

A total number $(N)$ of eight devices were tested, comparing the results obtained with PC fibers (PC, $N=4$ ) and NC devices (NC, $N=4$ ). Each test was conducted with GFP from individual blood donor.

\section{Analysis of PA}

At each time step (see Table 2), $200 \mu \mathrm{L}$ of circulating GFP was taken from the circuit, and the resulting platelet activation state (PAS) was measured as follows: from each sample, a $100-\mu \mathrm{L}$ tube containing (final concentrations) 5000 platelets/ $\mu \mathrm{L}, 200 \mathrm{nM}$ Ac-FIIa, $5 \mathrm{mM} \mathrm{Ca}^{2+}$, and $100 \mathrm{pM} \mathrm{FXa} \mathrm{was} \mathrm{incubated}$ at $37^{\circ} \mathrm{C}$ for $10 \mathrm{~min}$; then, a $10-\mu \mathrm{L}$ sample was assayed for thrombin generation using $0.3 \mathrm{mM}$ ChromozymTH (Tosyl-Gly-Pro-Arg-4-nitranilide acetate, Roche Life Science, Milano, Italy) as the thrombin-specific chromogenic peptide substrate in a 96-wells microplate reader (Multiskan GO, Thermo Fisher Scientific, Inc., Waltham, MA, USA). Kinetic absorbance readings were performed at room temperature at 405-nm wavelength for $8 \mathrm{~min}$ (6). The PAS value was calculated as the slope of the linear fitting of the absorbance time data points, over the 8-min kinetic reading. PAS values were normalized against those obtained by sonicating uncirculated platelets with a microprobe sonicator (HD 2070 Sonoplus, Bandelin

TABLE 1. Dimensions and materials of the elements composing the hydraulic circuit used for GFP flow loop circulation experiments

\begin{tabular}{|c|c|c|c|}
\hline Element & ID (inches) & Length $(\mathrm{cm})$ & Material \\
\hline Pump inset & $1 / 4$ & 55 & Raumedic ECC-Silicone \\
\hline Upstream tubing for hydraulic circuit* & $1 / 4$ & 65 & Dideco XS class VI-PVC \\
\hline Downstream tubing for hydraulic circuit ${ }^{\dagger}$ & $3 / 16$ & 40 & Dideco XS class VI-PVC \\
\hline Element & ID (inches) & $n$ & Material \\
\hline Tube-to-tube connector & $1 / 4-1 / 4$ & 2 & $\mathrm{PC}$ \\
\hline Tube-to-tube connector & $1 / 4-3 / 16$ & 2 & $\mathrm{PC}$ \\
\hline
\end{tabular}

\footnotetext{
*Upstream tubing connects the reservoir to the OXY inflow; ${ }^{\dagger}$ downstream tubing connects the OXY outflow to the reservoir. ID, internal diameter; $n$, number of elements; PC, polycarbonate; PVC, polyvinylchloride.
} 


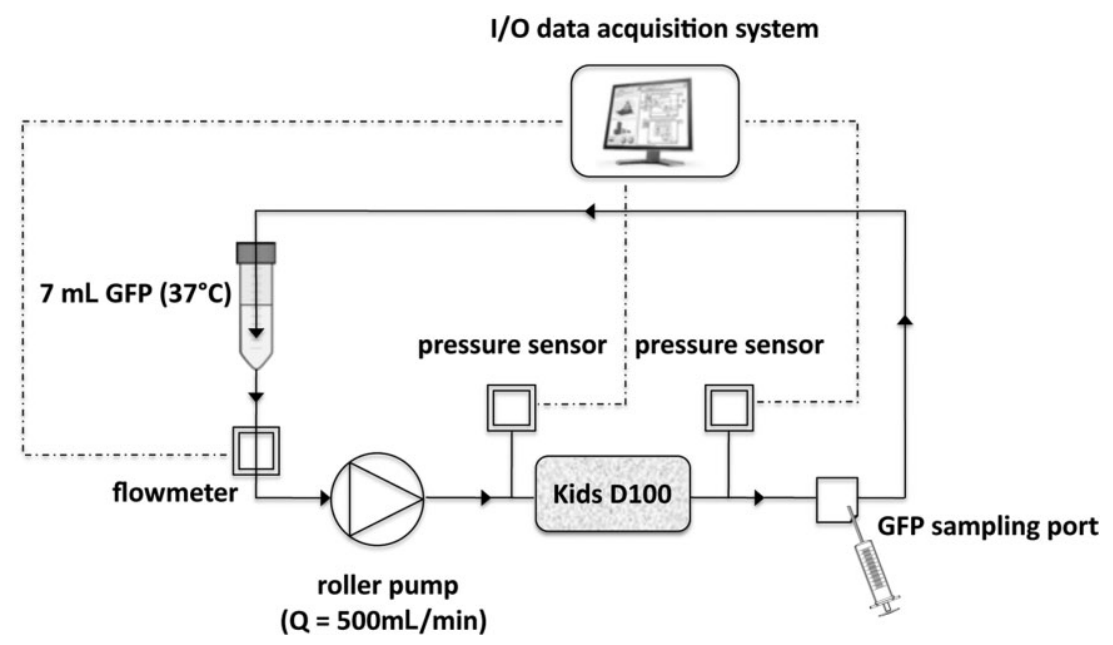

FIG. 3. Schematic of the experimental setup for GFP flow loop circulation experiments with the Kids D100 perfusion system: solid lines represent the hydraulic circuit with the arrows indicating the direction of flow; dashed lines indicate the electrical connections between the flow and pressure sensors and the $\mathrm{I} / \mathrm{O}$ data acquisition system.

Electronic GmbH \& Co. KG, Berlin, Germany). The sonication step is meant to yield platelets with maximal prothrombinase activity; thus, normalized PAS values (expressed in percentage, PAS [\%]) represent the bulk activity as a fraction of the thrombin generation rate of sonicated platelets $(100 \%)(22)$. Sonication conditions ( $10 \mathrm{~W}$ for $15 \mathrm{~s}$ ) were optimized for ovine platelets starting from the protocol reported in Schulz-Heik et al. (22) for human platelets. For each test, uncirculated platelets were also assayed, as the negative control. Change in PAS ( $\triangle \mathrm{PAS}$ ) was calculated over the duration of the circulation experiments $(0-120 \mathrm{~min})$, and the platelet activation rate (PAR) was quantified as the slope of the linear fitting of the PAS versus circulation time data points.

From each GFP sample, multiple spectophtometric readings $(n)$ were performed to evaluate data consistency; in detail: (i) for circulated platelets, at each time-point, two independent $100-\mu \mathrm{L}$ tubes were incubated, which were then split in two different microwells in the microplate reader, giving a number of readings, $n=4$; (ii) for sonicated samples, two independent GFP samples were sonicated, each incubated in two independent $100-\mu \mathrm{L}$

TABLE 2. Time points and corresponding number of passes at which GFP was taken from the flow loop setup and assayed via PAS assay

\begin{tabular}{lllrrrrr}
\hline Time (min) & $0 *$ & 10 & 20 & 30 & 60 & 90 & 120 \\
\# Passes & $0 *$ & 53 & 105 & 158 & 316 & 474 & 632 \\
\hline
\end{tabular}

\footnotetext{
*Indicates GFP sample taken after the circuit was filled and debubbled, before operating the pump.
}

tubes and split in two different microwells $(n=4)$; (iii) for blank samples, a $100-\mu \mathrm{L}$ tube was read in duplicate $(n=2)$ in the microplate reader.

\section{Statistical analysis}

Data of $\triangle \mathrm{P}, \triangle \mathrm{PAS}$, and PAR for the two groups (PC and NC fiber devices) are presented as mean \pm standard deviation (SD). The Shapiro-Wilk normality and $F$-test equality of variance tests were used to evaluate that the data were normally distributed; accordingly, results were compared through parametric Student's $t$-test. A $P$ value $<0.05$ was accepted as statistically significant.

\section{RESULTS}

The mean value of the $\Delta \mathrm{P}$ across the $\mathrm{OXY}$ remained constant for all the tested devices throughout the whole 120-min duration of the recirculation experiment: this evidence ensured that no undesirable fluid dynamic disturbances/anomalies affected the flowing GFP. Moreover, with equal rotational speed of the pump rotor $(1.85 \mathrm{rpm})$ set to deliver the GFP flow rate in the two sets of experiments, no statistically significant differences were observed comparing the $\Delta \mathrm{P}$ of the $\mathrm{PC}$ versus $\mathrm{NC}$ devices $(43.3 \pm 8.6$ vs. $46.3 \pm 5.9 \mathrm{~mm} \mathrm{Hg}, P=0.584)$. Those data indicate that in all the experiments, platelets were subjected to equivalent mechanical and fluidinduced solicitation offered by the pump propelling action and the streamflow within the hydraulic circuit and the device.

To discriminate between device-specific PA in response to the different surface membrane coating 


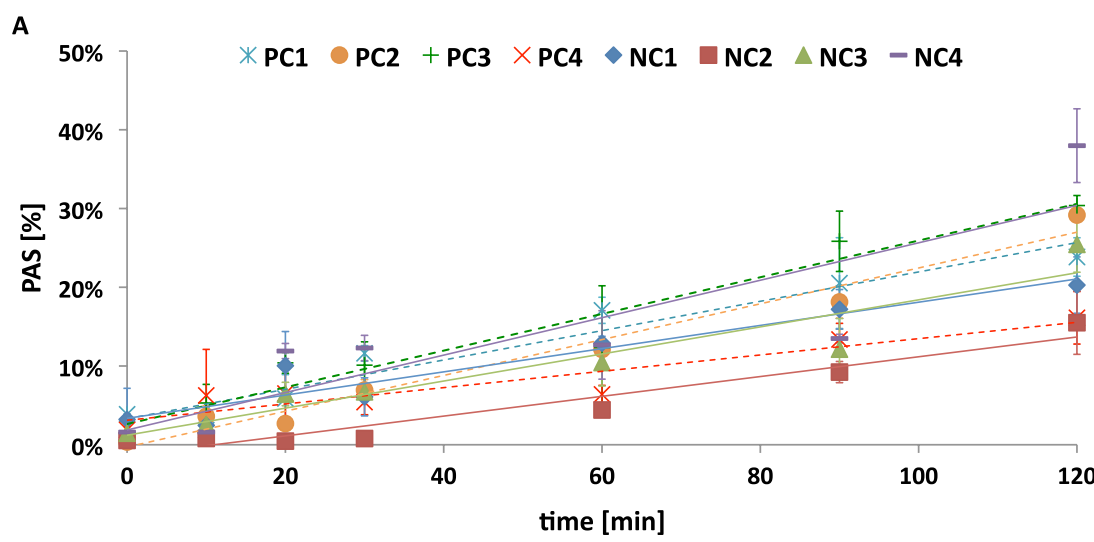

B

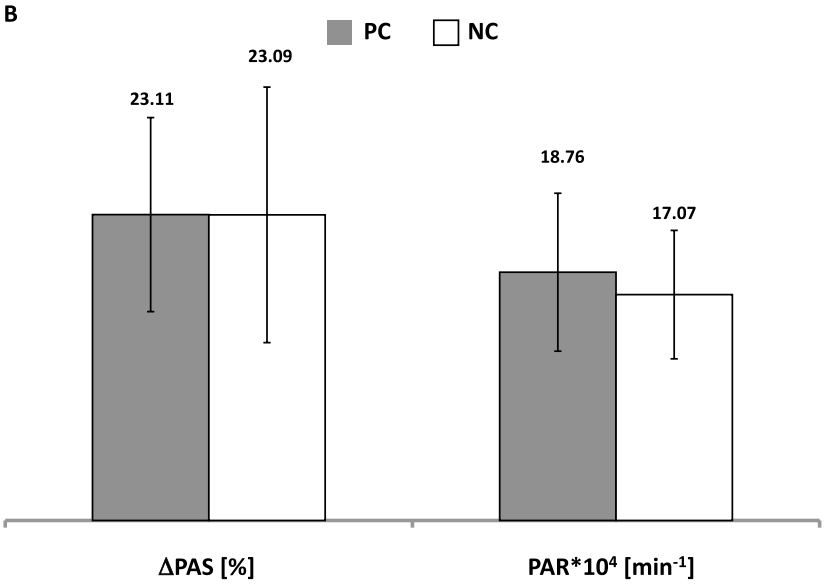

FIG. 4. (A) Normalized PAS values of recirculated GFP are plotted for PC and NC devices over the 120-min flow loop experiments (mean $\pm \mathrm{SD} ; n=4$ ): dashed and solid lines indicate the linear regression lines of the PAS versus circulation time data points, for the PC- and NC-devices, respectively; different colors identify each of tested devices (PC1-PC4 and NC1-NC-4). (B) Comparison of calculated $\triangle \mathrm{PAS}$ and PAR between $P C$ and $N C$ devices (mean $\pm S D$; for each group $N=4$ from independent blood donor). Data were normally distributed (Shapiro-Wilk normality test: $P=0.519$ and $P=0.859$ for $\triangle \mathrm{PAS}$ and PAR respectively; $F$-test equality of variance: $P=0.757$ and 0.756 for $\triangle$ PAS and PAR, respectively). Student's $t$-test revealed there is no statistically significant difference between the two groups ( $\triangle \mathrm{PAS}: P=0.601$; PAR: $P=0.356$ ). characterizing PC and NC devices, in Fig. 4, we report the results of PAS assay from the 120 -min in vitro circulation experiments. According to our data, a linear evolution of PA with increasing circulation time (i.e., number of passes) was observed for the GFP recirculated in both $\mathrm{PC}$ and $\mathrm{NC}$ devices (Fig. 4A). In addition, the comparison of PA between PC and NC devices (Fig. 4B) revealed that differences in $\triangle \mathrm{PAS}$ and PAR were not significant $\left(\Delta \mathrm{PAS} \mathrm{P}_{\mathrm{PC}}=23.09 \pm 9.66\right.$ vs. $\Delta \mathrm{PAS}_{\mathrm{NC}}=23.11 \pm 7.33$, $P=0.601 ; \mathrm{PAR}_{\mathrm{PC}}=17.07 \pm 4.85$ vs. $\mathrm{PAR}_{\mathrm{NC}}=18.76 \pm$ 5.97, $P=0.356)$, although interdonor variability had a notable influence on statistical dispersion of the data.

\section{DISCUSSION}

Thrombosis during $\mathrm{CPB}$ can be related to patientspecific factors and/or insufficient anticoagulation regimen. Nonetheless, thrombogenicity is potentiated by the nonphysiologic hemodynamic regimen associated to blood flow within medical BRDs $(2,24)$. Thus, a proper design of BRDs represents a key factor to be thoroughly investigated. Current criteria for the design of BRDs do not include the analysis of their associated thrombogenic potential. Typically, design specifications are focused on performance optimization, such as minimization of pressure losses and priming volumes, together with maximization of gas and/or heat transfer efficiency $(25,26)$. Flowinduced blood trauma is almost exclusively studied with respect to red blood cell damage (hemolysis). Thrombogenicity tests are performed on animal models for the preclinical evaluation of the device performance. However, animal studies are costly and time-consuming (24), and they are not compliant with ethics principles and with recent guidelines, which promote reducing the use of animals in research and testing (27). Moreover, in the context of a device's route toward clinical application, animal tests are typically performed at the late stage of the device design process. Although dealing with design changes (at any stage) in the design cycle is an inevitable reality for manufacturers, late stage design modifications can cause massive delays to the product design process and increment of manufacturing costs. Further, in vivo models are not even able to distinctively identify those specific design features that are responsible for device-related thrombogenicity. When preclinical results are not 
satisfactory, the general design of the device needs to be revised and tested iteratively, without any specific indication guiding the rational design optimization aimed at the minimization of the device thrombogenic potential.

In contrast, to date, different laboratory techniques, such as platelet aggregation testing, exist to estimate platelet function (28). Nevertheless, these laboratory tests are affected by severe limitations preventing these techniques to be widely accepted as valuable, standardized, and recognized methods for the thrombogenic optimization of BRDs. Major limitations include their inability of evaluating the initiation mechanism of $\mathrm{PA}$, that is, to discriminate between the influence of different agonists acting concomitantly, as occurring in ECC devices (mechanical and flow-induced solicitation together with surface contact-associated activation). In addition, they largely look at biochemical endpoints of PA rather than at its dynamics; thus, they are not suited for the analysis of dose and time PA rate, for characterizing recirculating ECC devices. These assays are performed using whole blood and/or platelet-rich plasma preparations as the platelet fluid vector, where the physiologic thrombin-mediated feedback activation mechanism further elevates the level of PA induced by the device. As a result, they are not reflective of the actual thrombogenic level of a given device, preventing the possibility of comparing the thrombogenic impact of different design solutions. Flow cytometry may be also applied for the study of PA dynamics using GFP preparations. However, flow cytometry implies repeated sample manipulation, required for fixation, antibody labeling, and washing, which may affect the accuracy of the analysis. Moreover, fixative agents (formaldehyde or paraformaldehyde) have been reported to potentially induce a decrease of the sample fluorescence intensity $(29,30)$, as well as a time-dependent modification of the PA level (31). Furthermore, flow cytometry requires expensive reagents (antibodies) and equipment (FACS) and highly specialized dedicated personnel. In the prospective of overcoming some of the limitations listed above and developing reliable procedures for the experimental evaluation of thrombogenicity in ECC medical devices, in this work, we designed an in vitro methodology able to characterize the dynamics of activation of platelets within BRDs, in experimental clinical-like flow conditions. Our investigation approach is based on the use of the PAS assay as the "tool" for characterizing PA dynamics. Notably, with the PAS assay, we can separate the different contributions concomitantly enhancing PA, selectively evaluate the flow- associated contribution on GFP which is related to the specific device geometry, and neglect all the other factors influencing and mediating thrombus formation in whole blood (i.e., plasma coagulation factors, thrombin-mediated PA feedback). As a result, the PAS assay can be employed for comparative analysis to identify a convenient design solution minimizing the thrombogenic potential.

In detail, our work was directed toward a twofold aim: (i) to test the efficacy of PAS assay to catch the dynamics of shear-dependent PA in response to repeated passes of platelets through a commercial hollow fiber blood oxygenator, where hemodynamic conditions are considerably different with respect to the MCS devices previously studied in literature; and (ii) to test the sensitivity of the PAS assay to surface contact-associated PA, comparing the platelet response induced by phosphorylcholine coating strategy in an unaltered fluid dynamic environment.

Among the variety of BRDs used for ECC, a neonatal OXY was chosen for our experimental campaign, even though it may not necessarily be the most thrombogenic device. The use of a neonatal perfusion system was dictated by the need to limit the blood volume to be retrieved from animals (a relatively large GFP volume is required for the flow loop). Furthermore, our choice was justified in consideration of the distinctive hemodynamics within the device, where different disturbed flow patterns arise, potentially eliciting PA, and of the considerable surface area and platelet residence time characterizing the hollow fiber bundle, enabling the analysis of the contact-induced platelet response. In fact, in the relatively complex blood flow path within a hollow fiber OXY device, flowing platelets are exposed to: (i) secondary flows and recirculation/ stagnation areas, such as those due to the gradual section reduction/enlargement of the inlet divergent and outlet convergent conduits, respectively; (ii) sudden changes of the velocity vectors (e.g., within the upstream and downstream manifolds of the inlet and outlet zones of the Kids D100) where the streamflow is driven circumferentially toward the hollow fiber bundle of the OXY module, and the outlet section, respectively [23]; (iii) anisotropic flow distribution across the array of the fiber bundle, where nonnegligible shear stress occurs due to the friction with the fiber walls, as reported in Pelosi et al. (32). In addition, there is supporting evidence in literature that phosphorylcholine surface coating is effective in protecting platelets from elevated activation during ECC (3,33-35). Thus, our experimental layout (comparison of PC vs. NC devices) allowed us to test the sensitivity of the assay to detect GFP- 
surface contact interactions under flow, in a convenient clinically pertinent in vitro setting.

According to our results, a linear dependency of the PA level with respect to the circulation time was detected, over a 120-min circulation flow loop. Those data provide evidence that the PAS assay is effective in detecting the dynamic of activation of recirculating platelets, as well as in quantifying even low changes in PA level, as the ones we observed at increasing number of passes. The linear dependency of PA with increasing time of exposure we observed does not support the platelet stimulation function defined by Boreda et al. (36), its key feature being the prediction that PA should vary linearly with shear stress but nonlinearly (as a fractional power) with time of exposure. Nonetheless, our results are in fair agreement with previous observations from GFP circulating flow loop experiments in severely disturbed dynamic flow conditions, as the ones generated in MCS $(6,13)$, as well as in in vitro stenotic tube-geometry systems with lower PA potential (37).

No significant differences have emerged by comparing PC versus $\mathrm{NC}$ devices, with comparable results obtained for $\triangle \mathrm{PAS}$ and PAR values. This represents the most intriguing aspect of our results. In fact, our data suggest that fluid shear-dependent contribution predominates the platelet response under flow and that, conversely, the effects of GFP-surface contact interactions are not detected, even in the presence of relevant surface area- and residence timecharacteristic devices. Similar observations have been recently reported by Merkle and colleagues, which described reduced deposition and adhesion of platelets on nanofiber composites under shear conditions with respect to static platelet incubation (38).

With material-surface interactions becoming not critical, the experimental methodology we developed allows focusing the evaluation of the device-induced thrombogenicity on local flow conditions deriving from specific device geometrical features, selectively. As for the PA values we found, our results do not directly correlate with in vivo clinical data (the present study was not directed toward the clinical characterization of the Kids D100). Firstly, our experiments were conducted with platelets of animal (ovine) origin. Although this choice was considered to be a reliable experimental approach, considering that pre-clinical trials of medical devices are typically performed with animal blood, limited interspecies differences emerged in terms of platelet thrombin production rate. According to our sonication experiments (data not shown), indeed, a prolonged exposure time $(15 \mathrm{~s})$ was required to maximize the prothrombinase activity of ovine platelets with respect to human platelets (10 s [22]), suggesting a relative different sensitivity to activation of platelets of ovine origin. Our results are supported by the study conducted by Tran and colleagues (39) who used the PAS assay to compare the sensitivity of human and ovine platelets to fluid shear stress, reporting few differences in the ovine platelet response. Moreover, our experiments do not account for the protective activation mechanism offered by administered antithrombotic drugs. In addition, our experiments are not reflective of the response of physiological whole blood, consisting of red and white blood cells and plasma proteins offering a complementary agonistic/antagonistic action on PA in vivo (40). In contrast, the use of isolated platelets (GFP) and the chemical modification of the prothrombin (acetylation) allowed us to examine the direct effect of fluid shear stress on PA, avoiding platelet aggregation and clot formation, and ensured that the thrombin produced by activated platelets, while assayable, did not further activate platelets. Lastly, clinical settings would not typically imply a number of passes of the patient blood through the Kids D100 perfusion system as high as 600 times, rather this was chosen as a reasonable target value in our GFP experiments as a means to amplify the detection of PA dynamics via the PAS assay in a 120 -min experiment.

Those discrepancies may limit the translational characteristic of our research. However, the proposed methodology provides an immediate and efficient in vitro approach for minimizing the shearinduced activation of platelets in BRDs. Remarkably, our study signals significant possibilities for the establishment of a reliable platform for the measurement and minimization of the PA potential in in vitro circulation models of cardiovascular ECC devices, as well as of different classes of hollow fiberrecirculating devices characterized by hemodynamic conditions potentially eliciting PA and thrombosis, such as blood detoxification systems and/or dialyzers.

\section{CONCLUSIONS}

This study shows that time-resolved activation of platelets induced by hemodynamic shear stress can be quantitatively assessed in blood recirculation device circulation flow loop platforms through the platelet activity state assay. It highlights that the PAS assay selectively focuses on the cumulative effects of shear stress and time of exposure on activation of platelets, while neglecting the role of surface contact phenomena. Accordingly, the PAS assay is a valuable and effective tool to support the design optimization of local flow dynamics in extracorporeal circulation 
devices in in vitro clinically pertinent perfusion conditions. Our methodology can be applied at any stage of the design optimization process, allowing characterization of the flow-dependent PA of a given (even not definitive) design solution, and comparing the influence of modifications of specific geometrical features conceived to reduce or eliminate the critical flow conditions that are responsible for eliciting PA and thrombosis. In contrast, in the clinical setting, patient's whole blood is recirculated during ECC, implying that additional aspects of hemostasis and thrombosis are involved, including surface contact PA. In view of this, we intend the proposed methodology as complementary to standard tests aimed at assessing the biocompatibility of materials used for blood handling medical devices.

Acknowledgments: The study was financially supported by 2011-2241 CARIPLO Foundation Grant. Project title: Evaluation of the thrombogenic risk: development of new approaches for the prediction of platelet activation and its minimization in artificial organs and prosthesis. The authors are grateful to SORIN Group Italia for supplying the oxygenator samples.

Conflict of Interest: Stefano Reggiani is an employee of Sorin Group Italia. For the remaining authors, none are declared.

\section{REFERENCES}

1. Ranucci M. Hemostatic and thrombotic issues in cardiac surgery. Semin Thromb Hemost 2015;41:84-90.

2. Ogawa S, Richardson JE, Sakai T, Ide M, Tanaka KA. High mortality associated with intracardiac and intrapulmonary thromboses after cardiopulmonary bypass. J Anesth 2011;26:919.

3. Pappalardo F, Della Valle P, Crescenzi G, et al. Phoshphorylcholine coating may limit thrombin formation during high-risk cardiac surgery: a randomized controlled trial. Ann Thorac Surg 2006;81:886-91.

4. van Veen JJ, Makris M. Management of peri-operative antithrombotic therapy. Anaesthesia 2015;70(S1):58-67.

5. Reynolds MM, Annich GM. The artificial endothelium. Organogenesis 2011;7:42-9.

6. Sheriff J, Girdhar G, Chiu WC, Jesty J, Slepian MJ, Bluestein D. Comparative efficacy of in vitro and in vivo metabolized aspirin in the DeBakey ventricular assist device. J Thromb Thrombolysis 2014;37:499-506.

7. Jesty J, Bluestein D. Acetylated prothrombin as a substrate in the measurement of the procoagulant activity of platelets: elimination of the feedback activation of platelets by thrombin. Anal Biochem 1999;272:64-70.

8. Yin W, Alemu Y, Affeld K, Jesty J, Bluestein D. Flow-induced platelet activation in bileaflet and monoleaflet mechanical heart valves. Ann Biomed Eng 2004:32:1058-66.

9. Nobili M, Sheriff J, Morbiducci U, Redaelli A, Bluestein D. Platelet activation due to hemodynamic shear stresses: damage accumulation model and comparison to in vitro measurements. ASAIO J 2008;54:64-72.
10. Alemu Y, Girdhar G, Xenos M, et al. Design optimization of a mechanical heart valve for reducing valve thrombogenicity: a case study with ATS valve. ASAIO J 2010;56:389-96.

11. Xenos M, Girdhar G, Alemu Y, et al. Device Thrombogenicity Emulator (DTE) design optimization methodology for cardiovascular devices: a study in two bileaflet MHV designs. $J$ Biomech 2010;43:2400-9.

12. Claiborne TE, Girdhar G, Gallocher-Lowe S, et al. Thrombogenic potential of Innovia polymer valves versus Carpentier-Edwards Perimount Magna aortic bioprosthetic valves. ASAIO J 2001;57:26-31.

13. Claiborne TE, Xenos M, Sheriff J, et al. Toward optimization of a novel trileaflet polymeric prosthetic heart valve via device thrombogenicity emulation. ASAIO J 2013;59:275-83.

14. Chiu WC, Girdhar G, Xenos M, et al. Thromboresistance comparison of the HeartMate II ventricular assist device with the device thrombogenicity emulation-optimized HeartAssist 5 VAD. J Biomech Eng 2014;36:021014.

15. Chiu WC, Slepian MJ, Bluestein D. Thrombus formation patterns in the HeartMate II ventricular assist device: clinical observations can be predicted by numerical simulations. ASAIO J 2014;60:237-40.

16. Marom G, Chiu WC, Crosby JR, et al. Numerical model of full-cardiac cycle hemodynamics in a total artificial heart and the effect of its size on platelet activation. J Cardiovasc Transl Res 2014;7:788-96.

17. Slepian MJ, Alemu Y, Girdhar G, et al. The Syncardia( $\left.{ }^{\mathrm{TM}}\right)$ total artificial heart: in vivo, in vitro, and computational modeling studies. J Biomech 2013;46:266-75.

18. Bluestein D. Towards optimization of the thrombogenic potential of blood recirculating cardiovascular devices using modeling approaches. Expert Rev Med Devices 2006;3:267-70.

19. Girdhar G, Bluestein D. Biological effects of dynamic shear stress in cardiovascular pathologies and devices. Expert Rev Med Devices 2008;5:167-81.

20. Bluestein D, Einav S, Slepian M. Device thrombogenicity emulation: a novel methodology for optimizing the thromboresistance of cardiovascular devices. J Biomech 2013;46:338-44.

21. Lawson DS, Smigla GR, McRobb CM, et al. A clinical evaluation of the Dideco Kids D100 neonatal oxygenator. Perfusion 2008;22:39-42.

22. Schulz-Heik K, Ramachandran J, Bluestein D, Jesty J. The extent of platelet activation under shear depends on platelet count: differential expression of anionic phospholipid and factor Va. Pathophysiol Haemost Thromb 2005;34:255-62.

23. Sorin Group-A Complete Family of Pediatric Perfusion Systems. 2010. Available at: http://www.sorin.com/file/view1559.action. Accessed January 3, 2015.

24. Lu Q, Hofferbert BV, Koo G, Malinauskas RA. In vitro shear stress-induced platelet activation: sensitivity of human and bovine blood. Artif Organs 2013;37:894-903.

25. Bhavsar SS, Schmitz-Rode T, Steinseifer U. Numerical modeling of anisotropic fiber bundle behavior in oxygenators. Artif Organs 2011;35:1095-102.

26. Consolo F, Fiore GB, Pelosi A, Reggiani S, Redaelli A. A numerical performance assessment of a commercial cardiopulmonary by-pass blood heat exchanger. Med Eng Phys 2015;37:584-92.

27. National Centre for the Replacement, Refinement \& Reduction of Animals in Research. The 3Rs. Available at: https:// www.nc3rs.org.uk/the-3rs. Accessed January 3, 2015.

28. Martin Y, Lépine M, Bannari A, Vermette P. Instrument and technique for the in vitro screening of platelet activation from whole blood samples. Rev Sci Instrum 2007;78:054302.

29. Hu H, Daleskog M, Li N. Influences of fixatives on flow cytometric measurements of platelet P-selectin expression and fibrinogen binding. Thromb Res 2000;100:161-6.

30. Schmidt V, Hilberg T, Franke G, Gläser D, Gabriel HH. Paraformaldehyde fixation induces a systematic activation of platelets. Platelets 2003;14:287-94. 
31. Atar OD, Eisert C, Pokov I, Serebruany VL. Stability validation of paraformaldehyde-fixed samples for the assessment of the platelet PECAM-1, P-selectin, and PAR-1 thrombin receptor by flow cytometry. $J$ Thromb Thrombolysis 2010;30:79-83.

32. Pelosi A, Sheriff J, Stevanella M, Fiore GB, Bluestein D, Redaelli A. Computational evaluation of the thrombogenic potential of a hollow-fiber oxygenator with integrated heat exchanger during extracorporeal circulation. Biomech Model Mechanobiol 2012;13:349-61.

33. Campbell EJ, O'Byrne V, Stratford PW, et al. Biocompatible surfaces using methacryloylphosphorylcholine laurylmethacrylate copolymer. ASAIO J 1994;40:M853-857.

34. Ishihara K, Fukumoto K, Iwasaki Y, Nakabayashi N. Modification of polysulfone with phospholipid polymer for improvement of the blood compatibility. Part 2. Protein adsorption and platelet adhesion. Biomaterials 1999;20:1553-9.

35. De Somer F, François K, van Oeveren W, et al. Phosphorylcholine coating of extracorporeal circuits provides natural protection against blood activation by the material surface. Eur J Cardiothorac Surg 2000;18:602-6.
36. Boreda R, Tatemi RS, Rittgers SE. Potential for platelet stimulation in critically stenosed carotid and coronary arteries. J Vasc Invest 1995;1:26-37.

37. Jesty J, Yin W, Perrotta P, Bluestein D. Platelet activation in a circulating flow loop: combined effects of shear stress and exposure time. Platelets 2003;14:143-9.

38. Merkle VM, Martin D, Hutchinson $M$, et al. Hemocompatibility of poly(vinyl alcohol)-gelatin core-shell electrospun nanofibers: a scaffold for modulating platelet deposition and activation. ACS Appl Mater Interfaces 2015;7:8302-12.

39. Tran PL, Merkle VM, DeCook T, et al. Platelet activity state in human, bovine and ovine species under constant shear stress: a comparative study. Proceedings of the ASME 2013 Summer Bioengineering Conference, SBC2013, June 26-29, Sunriver, Oregon, USA. 2013.

40. Ruggeri ZM, Mendolicchio GL. Adhesion mechanisms in platelet function. Circ Res 2007;100:1673-85. 